\title{
ESTILOS DE VIDA QUE PRACTICAN ESTUDIANTES DE LA ESCUELA UNIVERSITARIA DE CIENCIAS DE LA SALUD.
}

\author{
Lifestyles students practicing the school of health sciences. \\ *Vilma Mercedes Miranda Baquedano, **Daniela María Ayes Panchamé.
}

\section{RESUMEN}

Según la Organización Mundial de la Salud estilos de vida son "una forma general de vida basada en la interacción entre las condiciones de vida y los patrones individuales de conducta determinados por factores socioculturales y características personales. Objetivo Describir los estilos de vida que practican estudiantes de la Escuela Universitaria de Ciencias de la Salud en la Universidad Nacional Autónoma de Honduras en el Valle de Sula (EUCS-UNAHVS), durante el primer semestre del 2015. Pacientes y metodos Estudio cuantitativo descriptivo transversal. Población 3540, muestra 347 estratificando por carrera: Enfermería 98, Odontología 164 y Medicina 85. Instrumento tipo encuesta, previo consentimiento informado. Resultados EI 57\% (198) consumían alimentos en cafetería, 33\% (114) alimentos de casa y $10 \%$ (35) compraban en restaurantes. El 47\% (163) hacía ejercicio. El 28\% (97) consumía más de 8 vasos con agua diarios. 51\% (177) consumió bebidas alcohólicas. 30\% (104) consumió sustancias psicoactivas. $59 \%$ (205) algunas veces duermen mínimo 7 horas. Conclusión Los resultados señalan que los estudiantes del área de la salud practican parcialmente los estilos de vida saludables ejerciendo

\footnotetext{
*Profesora de la Carrera de Enfermería de la Escuela Universitaria de Ciencias de la salud (EUCS) de la Universidad Nacional Autónoma de Honduras en el Valle de Sula (UNAH - VS). Licenciada en Enfermería. Especialista en Salud Materno Perinatal. Magister en Gestión Educativa.

** Estudiante de la Carrera de Enfermería de la EUCS-UNAHVS.

algunas conductas de riesgo para su bienestar biopsicosocial.

\section{PALABRAS CLAVE}

Conductas saludables, Estilo de vida, Estudiantes del área de la salud.

\section{ABSTRACT}

According to the World Health Organization lifestyles are "a general way of life based on the interaction between living conditions and individual patterns of behavior determined by cultural factors and personal characteristics. Objetive: To describe the lifestyles that students practice University School of Health Sciences at the National Autonomous University of Honduras in the Valle de Sula (EUCS-UNAHVS), made the first half of 2015. Patients And Methods quantitative descriptive cross-sectional study Population 3540,347 sample stratified by race: 98 nursing, Dentistry and Medicine 85. 164 survey instrument type, prior informed consent. Results $57 \%$ (198) ate cafeteria food, $33 \%$ (114) food from home and 10\% (35) bought in restaurants. The $47 \%$ (163) itself to exercise. The $28 \%$ (97) consumed more than 8 glasses of water a day. $51 \%$ (177) consumed alcoholic beverages. 30\% (104) consumed psychoactive substances. 59\% (205) sometimes sleep at least seven hours. Conclusion The results indicate that students in the area of health practice partially healthy lifestyles, exercising some risk behaviors for biopsychosocial being. 


\section{KEYWORDS}

Health behavior, life style, students, health occupations.

\section{INTRODUCCION}

Estilos de vida saludable son todas aquellas conductas que tenemos asumidas como propias en nuestra vida y que intervienen positivamente en nuestro bienestar físico, mental y social, estos influyen en el estado de salud y no practicarlos se vincula con enfermedades.

La OMS recomienda que se adopte un estilo de vida saludable a lo largo de todo el ciclo vital, con el fin de preservar la vida, mantenerse sano y aminorar la discapacidad y el dolor en la vejez. ${ }^{(1)}$

Marc Lalonde citado por Gonzales describe en salud pública 4 determinantes para la salud: la biología humana, el medio ambiente, sistema de asistencia sanitaria y estilos de vida. ${ }^{(2)}$ Pero lo que más afecta la salud de las personas son los estilos de vida, ya que están estrechamente vinculados con el mantenimiento y mejoramiento de la salud.

Entre los factores que pueden influir a modificar los estilos de vida tenemos las condiciones generales como: socioeconómicas, culturales y ambientales; las condiciones de vida, e influencia social. ${ }^{(2)}$

En la vida de los estudiantes universitarios gran parte de estos factores están presentes en sus vidas y pueden causar modificaciones en sus estilos de vida.

Un estilo de vida saludable está integrado por un conjunto de factores que deben funcionar de manera armoniosa y completa: lo biológico, la herencia y el medio ambiente.(3)

\section{ESTILOS DE VIDA SALUDABLE}

Alimentación y Dieta: El alimento le brinda a nuestro cuerpo la energía que necesitamos para funcionar. Los hábitos alimentarios constituyen un factor determinante del estado de salud de una persona o una comunidad.

La salud y la vida de las personas dependen en gran parte de la calidad nutricional de los alimentos que consumen diariamente, la cual a su vez depende de la calidad higiénica y sanitaria a que estos son sometidos en toda la cadena productiva, desde el campo hasta la mesa del consumidor. ${ }^{(4)}$

Cada vez se dedica menos tiempo en la elaboración de los alimentos, prefiriéndose los alimentos procesados o el comprar comida conocida como comidas rápidas esto es más común en los jóvenes estudiantes. La dieta promedio cada vez es más abundante en grasas, dulces y carnes y carecen de vegetales; rehúyen a los métodos de cocción más saludables (al vapor) a favor de aquellos más pesados (las frituras) y prefieren los productos muy elaborados, congelados y recalentados a aquellos naturales. ${ }^{(5)}$ Entonces lo que verdaderamente hace el estudiante universitario es alimentarse, no nutrirse.

Actividad Física y diversión: Se refiere a la energía utilizada para el movimiento, se trata por tanto de un gasto de energía adicional del que necesita el organismo para mantener las funciones vitales. $\left.{ }^{6}\right)$

Las actividades cotidianas como por ejem- 
plo, el trabajo, las tareas del hogar, las actividades de ocio o de tiempo libre y el transporte la consideramos como actividad física. El término ejercicio hace referencia a movimientos diseñados y planificados específicamente para estar en forma y gozar de buena salud. ${ }^{(6)}$ Sin embargo el grado de sedentarismo se ha ido elevando en los últimos años y los jóvenes estudiantes no se quedan atrás en la disminución de la actividad de ejercicio que realizan. En los estudiantes son varios factores que pueden contribuir al poco ejercicio como son: el poco tiempo disponible, la delincuencia que está sufriendo el país y en cierta medida el nivel económico. El sedentarismo es una de las 10 causas fundamentales de mortalidad y discapacidad.

La OMS estima que la inactividad física causa en conjunto 1,9 millones de defunciones a nivel mundial y está relacionada con la prevalencia del cáncer de mama, cáncer colorectal, diabetes mellitus y aproximadamente un $22 \%$ de los casos de cardiopatía isquémica. ${ }^{(7)}$

Consumo de agua: El agua es el principal componente del ser humano, constituyendo entre 55 y $70 \%$ de su peso corporal. Dado que no se sintetiza a nivel endógeno, es necesario ingerirla, siendo por tanto un nutriente esencial. Se requiere una ingesta mínima de dos litros diarios. ${ }^{\left({ }^{8}\right)}$ Cumple diversas funciones en el organismo, sin embargo el consumo de agua ha sido desplazado por el consumo de bebidas azucaradas así como refrescos y jugos artificiales, por lo que el consumo de bebidas azucaradas está aso ciado con hábitos alimentarios pocos saludables así como con muchas otras enfermedades.
Sueño y Descanso: El sueño es un elemento central en la fisiología del ser humano, tener un patrón de sueño adecuado permite a los sujetos contribuir a una mejor calidad de vida, este patrón se ve modificado durante la vida universitaria.

La distribución promedio de horas necesarias para un adecuado estado de vigilia, se han descrito entre 7 a 9. La calidad de sueño, va más allá del número de horas dormidas; se refiere al hecho de dormir bien y continuamente durante la noche y tener un buen funcionamiento durante el día. La cantidad de sueño no sólo se ha relacionado con la salud física sino también con diversas medidas de bienestar psicológi$\mathrm{co}^{(9)}$

\section{ESTILOS DE VIDA NO SALUDABLES}

Consumo de sustancias nocivas: Las drogas psicoactivas son sustancias químicas que afectan los procesos mentales y el comportamiento porque cambian por períodos temporales la conciencia. En el encéfalo estas sustancias se unen a los receptores sinápticos y entorpecen o incitan ciertas reacciones, con ello alteran profundamente el sistema de comunicación encefálica y alteran la percepción, memoria, estado de ánimo y conducta. ${ }^{(10)}$

Las drogas psicoactivas se dividen en:

- Opiáceos o narcóticos: como la morfina y heroína.

- Alucinógenos: (fenciclidina éxtasis).

- Canabicos: marihuana

- Depresores: barbitúricos, benzodiacepinas, alcohol.

- Estimulantes: anfetaminas, metanfetaminas, cocaína, nicotina y cafeína. 
Tabaquismo: Su consumo es la epidemia que más mortalidad causa en el mundo ya que favorece la aparición de numerosas enfermedades, como cáncer de pulmón, cardiopatía isquémica, bronquitis crónica y enfisema, entre otras. ${ }^{(10)}$ Es importante considerar que el tabaco no es peligroso sólo para quien lo consume en forma de cigarrillo, sino para quienes inhalan las sustancias procedentes de él a través del humo del tabaco medioambiental.

Consumo de alcohol: El alcohol es una de las drogas más consumidas principalmente en actividades sociales y es aceptado como un acompañamiento placentero de las relaciones y los encuentros sociales y se ha extendido entre los jóvenes.

Entre los jóvenes, el alcohol es la droga predilecta. La población universitaria es de alta vulnerabilidad para el consumo de alcohol, de hecho, los adolescentes lo consumen con mayor frecuencia e intensidad que todas las demás drogas ilícitas combinadas, ${ }^{(11)}$ esto es debido a varios factores: individuales como búsqueda de libertad, autonomía e identidad, búsqueda de nuevas emociones y sensaciones, mecanismos de escape y por curiosidad.

Cafeína: La cafeína es un estimulante del sistema nervioso central que produce un efecto temporal de restauración del nivel de alerta y eliminación de la somnolencia. Las bebidas que contienen cafeína, tales como el café, el té, algunas bebidas no alcohólicas (especialmente los refrescos de cola) y las bebidas energéticas gozan de popularidad. La cafeína es la sustancia psicoactiva más ampliamente consumida en el mundo. ${ }^{(11)}$
Consumo de otras drogas: El consumo de drogas (ilegales) es uno de los problemas que mayor interés ha despertado en las últimas décadas debido a la creciente implicación de los adolescentes y jóvenes en estas conductas. Constituye un auténtico problema de salud pública y social debido a los costos humanos, sociales y económicos que conlleva. ${ }^{(12)}$

Para muchos jóvenes el consumo de drogas resulta como una alternativa para evadir sus problemas y satisfacer el deseo de prolongar e intensificar momentos agradables y placenteros, durante los cuales desaparece aparentemente la incertidumbre y el desencanto generalizado que los rodea.

El objetivo fue, describir los estilos de vida que practican los estudiantes de la Escuela de Ciencias de la salud en la Universidad Nacional Autónoma de Honduras en el Valle de Sula.

\section{MATERIALES Y MÉTODOS}

Se realizó un estudio cuantitativo de tipo descriptivo transversal para describir los estilos de vida que practican los estudiantes de la EUCS UNAH-VS. El universo fue 3540 estudiantes de las carreras de medicina (865), enfermería (994) y odontología $(1,681)$. Tomándose una muestra estratificada de 347 estudiantes: medicina 85, enfermería 98 y odontología 164. Se realizó una encuesta que contaba con 39 preguntas abiertas y cerradas la cual evaluó 5 categorías que nos permitieron identificar los estilos de vida saludables de los estudiantes, estas son: la caracterización de los estudiantes:

1. Nutrición: índice de masa corporal (IMC) con la siguiente formula $(\mathrm{kg} / \mathrm{mt} 2)$ y lugar 
donde adquirían los alimentos.

2. Actividad física y tiempo de ocio.

3. Consumo de agua.

4. Sueño y descanso.

5. Uso de sustancias nocivas.

La aplicación de la encuesta se llevó a cabo con los estudiantes que asistían por la tarde a clases, previo consentimiento informado.

Los datos fueron analizados utilizando el programa EPI INFO 3.5.4.

\section{RESULTADOS}

De los 347 jóvenes encuestados 88\% (307) tenían edades de 18 a 25 años, 8\% (27) entre 26 a 33, 3\% (10) más de 34 años y $1 \%$ (3) no contestó. Se encontró que $77 \%$ (267) eran mujeres y 23\% (80) hombres.

El 53\% (184) contaba con una jornada educativa mayor de 8 horas, 34\% (117) jornada de 6 a 8 horas y 13\% (46) de 4 a 6 horas. Se encontró que $75 \%$ (261) de los estudiantes conocían su peso/talla y $25 \%$ (86) lo desconocían. Con estos datos se les calculo el IMC. Ver Tabla No. 1

Tabla No. 1: Índice de masa corporal de los estudiantes de la EUCS.

\begin{tabular}{|lccc|}
\hline \multicolumn{1}{|c}{ IMC * } & Mujer & Hombre & $\%$ \\
\hline $\begin{array}{l}\text { DELGADEZ } \\
\text { ACEPTABLE }\end{array}$ & 20 & 1 & $6 \%$ \\
\hline $\begin{array}{l}\text { DELGADEZ } \\
\text { MODERADA }\end{array}$ & 2 & 0 & $0.6 \%$ \\
\hline OBESO TIPO 1 & 12 & 2 & $4 \%$ \\
\hline OBESO TIPO 2 & 1 & 0 & $0.3 \%$ \\
\hline OBESO TIPO 3 & 2 & 0 & $0.6 \%$ \\
\hline SOBRE PESO & 46 & 32 & $22.5 \%$ \\
\hline PESO NORMAL & 112 & 31 & $41 \%$ \\
\hline NO CONTESTO & 72 & 14 & $25 \%$ \\
\hline TOTAL & 267 & 80 & $100 \%$ \\
\hline
\end{tabular}

* Indice de masa corporal

Fuente: Encuesta Asignatura Salud Familiar 1
El 50\% (175) de los estudiantes encuestados se ven a sí mismos con buena salud, $33 \%$ (113) dijeron verse con salud regular, $12 \%$ (42) con excelente salud y $5 \%$ (17) con mala salud.

En cuanto al horario de consumo de alimentos, se encontró que: 55\% (190) algunas veces comen en horario específico, 30\% (106) no comen en horario establecido, 15\% (51) cumplen su horario de alimentación. El $57 \%$ (197) de los estudiantes consumían alimentos de cafetería. Ver Gráfica No. 1.

Gráfica No. 1: Fuente de los alimentos consumidos.

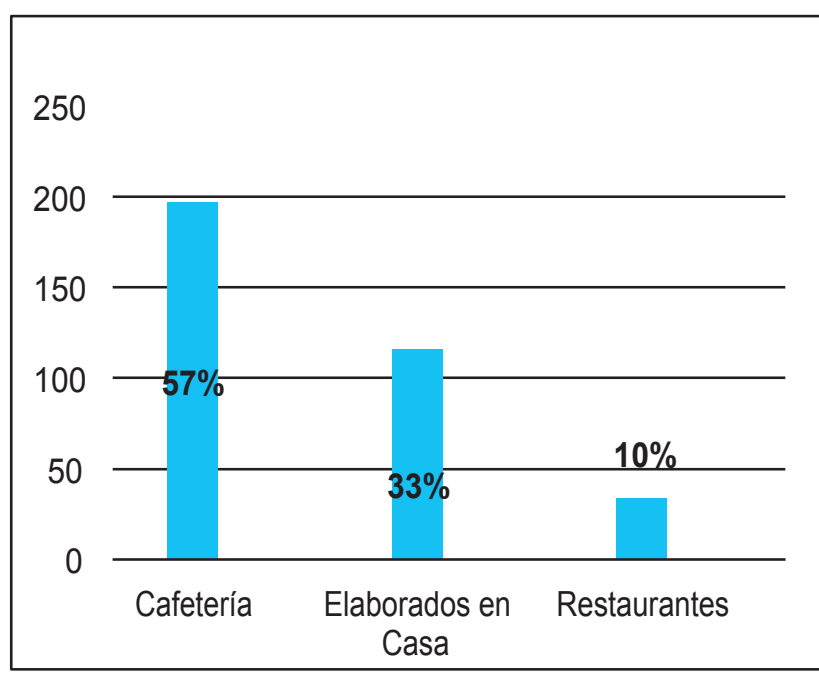

Fuente: Encuesta Asignatura Salud Familiar 1

Se encontró que 53\% (184) realizaba ejercicio físico y $47 \%$ (163) no practicaba esta actividad.

En cuanto al tiempo que los estudiantes disponen para actividades recreativas (ocio); 58\% (201) señaló que algunas veces destinan parte de su tiempo libre para actividades académicas, 40\% (137) que siempre destinan su tiempo de ocio para actividades académicas. Ver tabla No. 2. 
Tabla No. 2: Actividad que realiza en tiempo de ocio

\begin{tabular}{|lcr|}
\hline $\begin{array}{l}\text { Actividad que realiza en } \\
\text { tiempo de ocio }\end{array}$ & Número & Porcentaje \\
\hline $\begin{array}{l}\text { Algunas veces realizan } \\
\text { actividades académicas }\end{array}$ & 201 & $58 \%$ \\
\hline $\begin{array}{l}\text { Siempre realizan actividades } \\
\text { académicas }\end{array}$ & 137 & $40 \%$ \\
\hline $\begin{array}{l}\text { Nunca realizan actividades } \\
\text { académicas (lo invierten en } \\
\text { actividades recreativas }\end{array}$ & 9 & $2 \%$ \\
\hline $\begin{array}{l}\text { TOTAL } \\
\text { Fuente: Encuesta Asignatura Salud Familiar } 1\end{array}$ & 347 & $100 \%$ \\
\hline
\end{tabular}

Sobre el consumo de agua, $72 \%$ (250) respondió consumir menos de 8 vasos al día y $28 \%$ (97) más de 8 vasos con agua.

Al preguntarles sobre algunos hábitos no saludables, $13 \%$ (45) dijo haber consumido tabaco. (Ver tabla No. 3)

Tabla No. 3: Hábitos no saludables

\begin{tabular}{|lllr|}
\hline Hábitos & $\mathrm{Si}$ & No & TOTAL \\
\hline Tabaco & $45(13 \%)$ & $302(87 \%)$ & 347 \\
\hline Alcohol & $177(51 \%)$ & $170(49 \%)$ & 347 \\
\hline $\begin{array}{l}\text { Sustancias } \\
\text { psicoactivas }\end{array}$ & $73(21 \%)$ & $274(71 \%)$ & 347 \\
\hline
\end{tabular}

Fuente: Encuesta Asignatura Salud Familiar 1

Al consultarles si duermen 7 horas; las respuestas fueron:

$59 \%$ (204) contestaron que algunas veces $27 \%$ (94) que nunca y $14 \%$ (49) siempre.

\section{DISCUSION}

Los resultados de la investigación muestran que los estilos de vida de los futuros profesionales de la salud presentan en general deficiencias en las distintas variables evaluadas. En la alimentación y dieta únicamente $33 \%$ (116) de los estudiantes traen los alimentos de sus casas el resto lo compra en las cafeterias y restaurantes. En un estudio nutricional en estudiantes madrileños señala la preferencia de comprar comidas y que las golosinas son las preferida en las mujeres $26 \%$ y en los varones las comidas rapidas $31 \% .^{(13)}$

Al evaluar la nutrición por medio del IMC de los $261(75 \%)$ que conocían su peso y talla el $55 \%$ (143) estaban en un peso normal y el resto en sobrepeso y obesidad. A pesar de que fueron más mujeres que se encuestaron, la relación de obesidad es mayor en el hombre con $32(48 \%)$ de 66 hombres contra un $61(24 \%)$ de 261 mujeres. Lo que concuerda con un estudio en la Universidad de Madrid en donde el sobrepeso/obesidad es casi el doble en varones $(50 \%)$ frente a las mujeres $(28,6 \%)^{(14)}$

La universidad es un contexto ideal para aprender y consolidar la vida personal y profesional, incluyendo un estilo de vida que va a determinar la salud futura, sin embargo estos estilos de vida se van deteriorando debido a la participación de la mayor parte del tiempo en actividades académicas y no contar con el tiempo suficiente para la participación de actividades físicas y recreativas.

En esta investigación los resultados mostraron que $53 \%$ de los estudiantes pasan más de 8 horas al día en la universidad por lo que tienen una jornada completa y que $53 \%$ no realizan actividad física. Investigaciones han informado que la inactividad física es un problema grave entre los estudiantes universitarios. Comparándose con un estudio realizado en la Universidad Pontificia de Chile sobre el ejercico físico el $42,7 \%$ del grupo masculino y el $29,8 \%$ del grupo femenino responden que si la realizan. ${ }^{(15)}$ 
En esta investigación se encontró que $72 \%$ (250) de los estudiantes de la EUCS-UNAHVS ingieren menos de 8 vasos de agua al día, existiendo una brecha al compararlo con un estudio en España realizado por el Instituto Nacional de estadística en universi dades públicas y privadas con una muestra de 900 estudiantes y los datos mostraron que $86 \%$ de los estudiantes ingieren más de 8 vasos de agua natural al día. ${ }^{(13)}$

Más del $50 \%$ de los los estudiantes de la EUCS-UNAHVS duermen menos de 7 horas y consideran que no duermen bien lo que concuerdan con un estudio realizado en estudiantes universitarios Colombianos que solo duermen 5.5 horas diarias ${ }^{(16)}$ Las horas necesarias para una buena salud son de 7 a 8 y dormir menos de 7 horas aumenta el riesgo de muerte temprana y de diversas enfermedades; así como el aumento del consumo de café, alcohol y otras sustancias.

Dentro de los hábitos no saludables que practican los estudiantes se encontró que $21 \%$ consumían sustancias psicoactivas siendo las más frecuentes las bebidas energizantes, a diferencia de los resultados obtenidos en un estudio realizado en estudiantes de Tabasco en México, donde $76 \%$ de los encuestados aceptó haber consumido bebidas energizantes. ${ }^{(17)}$ Estos estudiantes refieren que ingirieron bebidas energéticas sobre todo en horas de estudio o durante los períodos de exámenes, aunque el $16 \%$ señaló que las consume porque le son agradables. El $21 \%$ de los encuestados dijo haber tenido uno o más efectos adversos. ${ }^{(17)}$

Otro de los hábitos no saludables es el con sumo de alcohol, que es practicado por $51 \%$ de los estudiantes que participaron en esta investigación. Dato que es superior a los reportados por una universidad privada de Colombia donde se encontró una prevalencia de alcoholismo del 23\%. ${ }^{(18)}$ Teniendo en cuenta las consecuencias del consumo de alcohol y las estadísticas sobre su uso y abuso en jóvenes, se convierte en un problema de salud pública y pone en evidencia la necesidad de ubicarlo como un tema de intervención contínua para los contextos universitarios. ${ }^{(19)}$

\section{CONCLUSION}

Los resultados señalan que los estudiantes del área de la salud practican parcialmente estilos de vida saludable, ejerciendo algunas conductas de riesgo para su bienestar biopsicosocial.

A diferencia de la población general, los profesionales de la salud que no lleven un estilo de vida saludable, representan un problema adicional en salud pública, no solo para su salud personal, sino para la de sus pacientes ya que se espera que sean el ejemplo de una población absorbida por las tendencias de la mala nutrición y el sedentarismo principales causas de los problemas en salud publica.

\section{RECOMENDACIÓN}

Impulsar desde la EUCS-UNAHVS estilos de vida saludable dentro de toda su población tanto estudiantes como docentes y para ser ejemplo dentro y fuera de la UNAH-VS considerando que se deben incorporar mayores áreas recreativas y espacios para ejercicos físicos y convertir la universidad no solo en espacios educativos sino también en entornos saludables. 


\section{BIBLIOGRAFÍA}

1. OMS. La buena salud añade vida a los años: información general para el Día Mundial de la Salud. 2012. [citado el 12 de Abril del 2015] Disponible en: http:// www.who.int/ageing/publications/whd 2012_global_brief/es/

2. González M.E Intervención de la universidad en la promoción de la salud de sus estudiantes. Tesis de doctorado. Universidade de Santiago de Compostela. 2009. ( citado el 20 de Mayo del 2015) Disponible en: https://dspace.usc. es/bitstream/10347/4991/1/pg_247-262 _innovacion19.pdf

3. Rodés J, Piqué JM, Trilla A. Libro de la salud del Hospital Clínica de Barcelona y la Fundación BBVA. Bilbao: Fundación BBA; 2007. [citado el 13 de Abril del 2015] Disponible en: https://books. google.hn/books?id=SsMyl7M0nZYC\&pg= PA65\&dq=factores + condicionantes + de+los+estilos+de+vida\&hl=es-419\&sa $=X \&$ ei=NDVMVdf5BsqhgwSj24CwDg\& ved=0CBsQ6Ahttps://books.google. $\mathrm{hn} /$ books?id=SsMyl7M0nZYC\&pg=PA65 $\& d q=$ factores + condicionantes $+d e+$ los+estilos + de + vida\&hl=es $-419 \&$ sa $=$ $X \&$ ei=NDVMVdf5BsqhgwSj24CwDg $\&$ ved $=0 C B s Q 6 A E w A A \# v=$ onepage $\& q=$ factores $\% 20$ condicionantes $\% 20$ de $\% 20$ los $\% 20$ estilos $\% 20$ de $\% 20$ vida $\& \mathrm{f}=\mathrm{false}$

4. FAO. Informes técnicos sobre ingeniería agrícola y alimentaria; 2009. [Actualizado 2015; citado el 12 de Mayo del 2015]
Disponible en: http://www.fao.org/3/a-i0 480s.pdf

5. Ediciones LEA S.A. Sobrepeso y obesidad: Terapias naturales para la curación integral. Medicinas Alternativas. Buenos Aires Argentina: Ediciones LEA; 2014. [citado el 15 de Marzo 2015] Disponible en: https://books.google.hn/books?id=C $z$ WZAwAAQBAJ\&printsec $=$ frontcover $\& d q=S o b r e p e s o+y+o b e s i d a d:+T e r a p i a s$ +naturales+para+la+curaci\%C3\%B3n+ integral\&hl=es-419\&sa $=X \& v e d=0 C B w$ Q6AEwAGoVChMI7aflsouBxwIViBse ChOTIQZe\#v=onepage \&q=Sobrepeso $\% 20 y \% 20$ obesidad\%3A\%20Terapias $\% 20$ naturales $\% 20$ para $\% 201 a \% 20$ curaci $\%$ C3\%B3n\%20integral\&f=false

6. Márquez RS. y Garatachea VN, Funiber. Actividad física y salud. Madrid: Ediciones Díaz de Santos; 2013. [Citado el 13 de Abril del 2015]. Disponible en: https: //books.google.hn/books?id=sxZr7nS2n $8 \mathrm{C} \&$ printsec $=$ frontcover\&dq=Activi $\mathrm{dad}+\mathrm{f}$ $\%$ C3\%ADsica+y+salud,+sara+marquez +rosa\&hl=es-419\&sa=X\&ved=0CBwQ6 wEwAGoVChMIltKTpliBxwIVBVYeCh00 CwC_\#v=one page\&q\&f=fal se

7. OMS. [Internet] Actividad Física. 2015. [citado el 30 de Marzo del 2015] Disponible en http://www.who.int/dietphysica lactivity/pa/es/

8. Royo Bordonada MA, Instituto de Salud Carlos III, Ministerio de Sanidad y Consumo, Escuela Nacional de Sanidad. 
Nutrición en Salud Pública. Madrid: Edita: Instituto de Salud Carlos III Ministerio de Sanidad y Consumo; 2007. [citado el 3 de Mayo 2015]. Disponible en: http://www.isciii.es/ISCIII/es/conte nidos/fd-publicaciones-isciii/fd-docu mentos/Nutricion_en_SP.pdf

9. Borquez P. Sleep quality, daytime sleepiness and self-perceived health university students. Universidad Nacional de Asunción (Paraguay). 2011 [Serie en internet]; 8(1):80-91. Disponible en: http://pepsic.bvsalud.org/pdf/eureka/ v8n1/a09.pdf

10. Gerrig RJ, Zimbardo PG. Psicología y Vida. 17a Ed. Pearson Educación: México. 2005 [citado el 4 Mayo del 2015] Disponible en: https://books.goo gle.hn/books?id=3-14Z1dAxo0C\&pg= $P A 163 \& l p g=P A 163 \& d q=\# v=$ onepage $\& q$ $\& f=$ false

11. Salcedo A., Palacios Espinosa X., y Espinosa A.F. Consumo de alcohol en jóvenes universitarios. Avances en Psicología Latinoamericana [Serial en internet] 2011; 29(1). 77-97. Disponible en: http://revistas.urosario.edu.co/index. php/apl/article/downloadSuppFile/ 640/25/

12. Gerónimo M, Mendieta M, Moccia A L. Opinión acerca de las condiciones de trabajo y estilo de vida en el área de Enfermería. 2014 [citado el 22 mayo del 2015]. Disponible en: http://www.enfer meria.fcm.unc.edu.ar/biblioteca/tesis/ geronimo_maria.pdf
13. Iglesias MT, Mata G, Pérez A, Hernández S, García-Chico R, Papadaki C. Estudio nutricional en un grupo de estudiantes universitarios madrileños. Nutrición clínica y Dietética Hospitalaria. [Internet] 2013 [citado el 11 Dic del 2015]; 33(1):23-30. Disponible en http://www. nutricion.org/publicaciones/revista 2013_33_01/ESTUDIO-NUTRICIONAL. pdf

14. Espinosa AF, Palacios Espinosa $X$, Salcedo Monsalve A. Consumo de alcohol en jóvenes universitarios. Avances en Psicología Latinoamericana. [Internet] 2011[Citado 15 de abril 2015]; 29(1). 77-97. Disponible en: http:// www.redalyc.org/pdf/799/79920065007. pdf

15. Bennasar M. Estilos de vida y salud en estudiantes universitarios: la universidad como entorno promotor de la salud. Tesis Doctoral Universidad de Les llles Dalears. [Citado el 2 Abril del 2015]. Disponible en: http://www.academia. edu/6223906/Estilos_de_vida_y_salud _en_estudiantes_universitarios_la_uni versidad_como_entorno_promotor_de_ la_salud._Miguel_Bennasar_Veny

16. Espinoza O Luis, Rodríguez R Fernando, Gálvez C Jorge, MacMillan K Norman. Hábitos de alimentación y actividad física en estudiantes universitarios. Rev. chil. nutr. [Internet]. 2011 Dic [citado 15 diciembre 2015 ] ; 38( 4 ): 458-465. Disponible en: http://www.scielo.cl/scielo. php? script =sci_arttext\&pid= S0717-75182011000400009\&Ing=es. 
http://dx.doi.org/10.4067/S0717-751820 11000400009.

17. Salvador DM, Cámara Flores J M, Cabral León FJ, Juárez Rojop IE, Díaz Zagoya JC. Consumo de bebidas energéticas en una población de estudiantes universitarios del estado de Tabasco.

Salud en Tabasco. [Internet] 2013[citado el 28 de Diciembre del 2015];19(1):10-14 Disponible en http://www.redalyc.org/pdf/ 487/48727474003.pdf

18. Ruiz ME, Del Pozo de la Calle S, Valero GT, Avila Torres JM, Varela MG. Habitos alimenticios y estilos e vida de los univer- sitarios españoles. Fundación Española de la Nutrición, Universidad CEU Madrid. [Citado el 27 de diciembre del 2015] Disponible en http://www.munideporte. com/imagenes/documentacion/ficheros/ 00DA422F.pdf

19. Arguello M, Bautista $Y$, Carbajal J, De Castro K, Díaz D, Escobar M, et. al. Estilos de Vida en Estudiantes del Area de la salud de Bucaramanga. Revista de Psicología. Rev de Psicología. [Internet] 2009[citado el 30 de Noviembre del 2015] ;1(2):27-41. Disponible en http:// pepsic.bvsalud.org/pdf/rpsua/v1n2/v 1n2a3.pdf 